Pacific Journal of Mathematics

REMARKS ON THE DIFFERENTIAL ENVELOPES OF 


\title{
REMARKS ON THE DIFFERENTIAL ENVELOPES OF ASSOCIATIVE ALGEBRAS
}

\author{
R. Coquereaux and D. Kastler \\ Dedicated to the memory of Henry Dye
}

\begin{abstract}
We study the relationship between the two types of differential envelopes of $Z$ /2-graded associative complex algebras; and describe the differential envelopes of a $Z / 2$-graded algebra as a simple modification of those of the associated ungraded algebra.
\end{abstract}

In this note we describe some aspects of the two existing notions of differential envelope of a $\mathbf{Z} / 2$-graded complex (or, for that matter real) associative algebra. Both notions are basic in the non commutative geometry under current development.

In $\S 1$, which fixes notation, we recall the definitions and main properties of the two types of differential envelope (= universal differential algebra) one attaches to a $\mathbf{Z} / 2$-graded algebra $A$. The first, $\tilde{\Omega}(A)=\mathbf{C} \tilde{\mathbf{1}} \oplus \Omega(A)$, pertains to general algebras (unital or not), and has a differential which vanishes nowhere on $A$ (in particular, if $A$ is unital with unit $\mathbf{1}, d \mathbf{1} \neq 0$ ). The second, $\Omega A$, is defined only for unital algebras, and has a differential vanishing on the unit. $\tilde{\Omega}(A)$ can accordingly be directly defined in terms of tensors over $A$, whilst the construction of $\Omega A$ uses either tensors over an $A$-bimodule, or a subfamily of tensors over $A$. Both $\Omega(A)$ (for that matter $\Omega(A)$ ) and $\Omega A$ are universal objects (= tied up with functors), the first in the general and the second in the unital category.

In $\S 2$ we discuss the relationship between the two notions, each of which is definable in terms of the other. On the one hand one has $\tilde{\Omega}(A)=\Omega \tilde{A}, \tilde{A}=\mathbf{C} \tilde{\mathbf{1}} \oplus A$ the augmented (Z/2-graded) algebra. On the other hand there is an injective multiplicative linear map of $\Omega A$ into the right ideal $A \tilde{\Omega}(A)$ of $\tilde{\Omega}(A)$, allowing one to consider $\Omega A$ as a linear subspace of tensors over $A$ (vanishing under the "consecutive diagonal mappings", and multiplying under "concatenation").

In $\S 3$ we show how the differential envelopes of a $\mathbf{Z} / 2$-graded algebra $A$ can be constructed in terms of those of the associated ungraded algebra (merely through a simple modification of the latter's product). 
This result is important from the algorithmic point of view, since it leads to a reformulation yielding formulae in which the "grading signs" occurs in a more transparent way (important in particular with respect to the algorithmics of $\mathbf{Z} / 2$-graded cyclic cohomology).

1. The two types of differential envelopes of an associative complex algebra. For the results quoted in this section we refer to [1] through [5]. In the sequel $A=A^{0} \oplus A^{1}$ throughout denotes a $\mathrm{Z} / 2$-graded complex algebra ${ }^{1}$, with set $A^{\cdot}=A^{0} \cup A^{1}$ of homogeneous elements ${ }^{2}$, and grading denoted by $\partial\left(\partial a=0\right.$ resp. $1(\bmod 2)$ for $a \in A^{0}$, resp. $\left.a \in A^{1}\right)$. $\tilde{A}$ denotes the augmented $\mathbf{Z} / 2$-graded algebra ${ }^{3}$, with unit $\tilde{\mathbf{1}}$ (irrespective of whether $A$ possesses a unit of its own or not-if this is the case, this unit is denoted by $\mathbf{1}$ ).

1.1. The first kind of differential envelope: the differential algebras $(\tilde{\Omega}(A), d)$ and $(\Omega(A), d)$. Let $A$ be a $\mathbf{Z} / 2$-graded algebra (not necessarily unital). We define, as an $\mathbf{N}$-graded vector space

$$
\tilde{\Omega}(A)=\sum_{n \in \mathbf{N}}^{\oplus} \tilde{\Omega}(A)^{n}
$$

where

$$
\tilde{\Omega}(A)^{n}=\tilde{A} \otimes A^{\otimes n}, \quad n \in \mathbf{N}
$$

with $\tilde{\Omega}(A)^{0}=\tilde{A}$. The intrinsic grading $\partial_{0}$ is defined by setting, for $a_{0} \otimes a_{1} \otimes \cdots \otimes a_{n} \in \Omega(A)^{n}$ :

$$
\partial_{0}\left(a_{0} \otimes a_{1} \otimes \cdots \otimes a_{n}\right)=\sum_{k=0}^{n} \partial a_{k},
$$

the total grading $\partial$ is then the sum of the intrinsic and the $\mathbf{N}$-grading. $\tilde{\Omega}(A)^{n}$ is made into a $\partial_{0}$-graded unital $\tilde{A}$-bimodule, by setting, for $a_{0}, x=\lambda \tilde{\mathbf{1}}+\underline{x} \in \tilde{A}^{*}, \lambda \in \mathbf{C}, a_{1}, \ldots, a_{n} \in A^{*}:$

$$
x\left(a_{0} \otimes a_{1} \otimes \cdots \otimes a_{n}\right)=x a_{0} \otimes a_{1} \otimes \cdots \otimes a_{n}
$$

\footnotetext{
I "Algebra" always means "complex associative algebra" (whereby "complex" could throughout be replaced by "real", changing $\mathbf{C}$ to $\mathbf{R}$ ).

${ }^{2}$ Generally, we denote by $E^{*}$ the set $E^{0} \cup E^{1}$ of homogeneous elements of any $\mathbf{Z} / 2$-graded vector space $E=E^{0} \oplus E^{1}$.

${ }^{3} \tilde{A}=\mathbf{C} \oplus A$ with product $(\lambda, a)(\mu, b)=(\lambda \mu, \lambda b+\mu a+a b), \lambda, \mu \in \mathbf{C}, a, b \in A$; and $\mathbf{Z} / 2$-grading $\tilde{A}^{0}=\mathbf{C} \oplus A^{0}, \tilde{A}^{1}=0 \oplus A^{1}$. The "added unit" is $\tilde{\mathbf{1}}=(1,0)$ (thus $\left.\tilde{A}=\mathbf{C} \tilde{\mathbf{i}} \oplus A\right)$. If $A$ is unital with unit $\mathbf{1}$, it appears as the ideal $(0, A)$ of $\tilde{A}$ generated by the idempotent $(0, \mathbf{1})$.
} 
and $^{4}$

$(1.5)\left(a_{0} \otimes a_{1} \otimes \cdots \otimes a_{n}\right) x$

$$
=\sum_{i=0}^{n-1}(-1)^{n+i+\sum_{k=t+1}^{n} \partial a_{k}} a_{0} \otimes \cdots \otimes a_{i} a_{i+1} \otimes \cdots \otimes a_{n} \otimes \underline{x}
$$$$
+a_{0} \otimes a_{1} \otimes \cdots \otimes a_{n} x \text {. }
$$

Defining then the product of $a_{0} \otimes a_{1} \otimes \cdots \otimes a_{n} \in \tilde{\Omega}(A)^{n}$ and $b_{0} \otimes b_{1} \otimes$ $\cdots \otimes b_{m} \in \tilde{\Omega}(A)^{m}$ as

$$
\begin{aligned}
& \left(a_{0} \otimes a_{1} \otimes \cdots \otimes a_{n}\right)\left(b_{0} \otimes b_{1} \otimes \cdots \otimes b_{n}\right) \\
& \quad=\left\{\left(a_{0} \otimes a_{1} \otimes \cdots \otimes a_{n}\right) b_{0}\right\} \otimes b_{1} \otimes \cdots \otimes b_{n},
\end{aligned}
$$

$\tilde{\Omega}(A)$ becomes an associative complex algebra, graded for both $\mathbf{Z} / 2$ gradings $\partial_{0}$ and $\partial$, and for the $\mathbf{N}$-grading.

Setting now, for $a_{0}=\lambda \tilde{\mathbf{1}}+\underline{a}_{0} \in \tilde{A}, \underline{a}_{0}, a_{1}, \ldots, a_{n} \in A$

$$
d\left(a_{0} \otimes a_{1} \otimes \cdots \otimes a_{n}\right)=\tilde{\mathbf{1}} \otimes \underline{a}_{0} \otimes a_{1} \otimes \cdots \otimes a_{n}
$$

specifies $d$ as a linear operator of $\tilde{\Omega}(A)$, of $\mathbf{N}$ - and $\partial$-grade 1 , and of vanishing square, which fulfills the graded derivation property

$$
\left\{\begin{array}{l}
d(\omega \psi)=(d \omega) \psi+(-1)^{\partial \omega} \omega d \psi, \\
\omega, \psi \in \tilde{\Omega}(A), \omega \text { of total grade } \partial \omega,
\end{array}\right.
$$

thereby making $\tilde{\Omega}(A)$ a $\partial$-graded (in fact bigraded $^{5}$ differential algebra): elements of $\tilde{\Omega}(A)$ are then reinterpreted as follows:

$$
a_{0} \otimes a_{1} \otimes \cdots \otimes a_{n}=a_{0} d a_{1} \cdots d a_{n} .
$$

It is useful to note that

$$
\tilde{\Omega}(A)=\mathbf{C} \tilde{\mathbf{1}} \oplus \Omega(A)
$$

where $\Omega(A)$, the direct sum of the

$$
\Omega(A)^{n}= \begin{cases}A, & n=0, \\ \tilde{\Omega}(A)^{n}, & n \geq 1\end{cases}
$$

is an ideal of $\tilde{\Omega}(A)$ decomposing in turn as

$$
\Omega(A)=d \Omega(A) \oplus A \Omega(A)
$$

${ }^{4}$ This formula becomes transparent by identifying the l.h.s. with $\left(a_{0} d_{1} \cdots d a_{n}\right) x$ (cf. (1.9) below), and transforming the latter expression using the graded derivation property of $d$ (cf. (1.8) and (1.9) below).

${ }^{5} \mathrm{a} \mathbf{Z} / 2$-graded differential algebra $\left(\Xi=\Xi^{+} \oplus \Xi^{-}, \delta\right)$ is a bigraded differential algebra whenever the algebra $\Xi$ has $\mathbf{N}$-grading $\Xi=\sum_{n \in \mathbf{N}}^{\oplus} \Xi^{n}$ such that (i) $\Xi^{n}=\Xi^{n} \cap \Xi^{+} \oplus \Xi^{n} \cap \Xi^{-}, n \in \mathbf{N}$ and $\delta\left(\Xi^{n}\right) \subset \Xi^{n+1}, n \in \mathbf{N}$. 
with $A \Omega(A)$ a right ideal of $\Omega(A)$ (generated by the unit 1 of $A$ for a unital $A)$.

$\Omega(A)$ is a universal object, which could more transparently (but less explicitly) be defined as the quotient of the free algebra of the set $\{a, d a ; a \in A\}$ through the ideal generated by ${ }^{6}$

$$
\begin{cases}\lambda \cdot a+\mu \cdot b \dot{-}(\lambda a+\mu b), & \\
\lambda \cdot d a \dot{+} \mu \cdot d b \dot{-}(\lambda a+\mu b), & \left\{\begin{array}{l}
a, b \in A, \\
\lambda, \mu \in \mathbf{C},
\end{array}\right.\end{cases}
$$

As a consequence ${ }^{7}$, given a $Z / 2$-graded complex algebra $B$, and a pair $(\alpha, \Delta)$ of a homomorphism ${ }^{8} \alpha: A \rightarrow B$ and a graded $\alpha$-derivation $\Delta: A \rightarrow B$ (i.e. a grade one linear map fulfilling

$$
\Delta(a b)=(\Delta a) \alpha(b)+(-1)^{\partial a} \alpha(a) \Delta b, \quad\left\{\begin{array}{l}
a \in A, \\
b \in A,
\end{array}\right)
$$

there is a unique homomorphism ${ }^{8} \theta: \Omega(A) \rightarrow B$ such that

$$
\left\{\begin{array}{l}
\theta(a)=\alpha(a), \\
\theta(d a)=\Delta a,
\end{array}, a \in A,\right.
$$

specifically given by

$$
\left\{\begin{array}{l}
\theta\left(a_{0} d a_{1} \cdots d a_{n}\right)=\alpha\left(a_{0}\right) \Delta a_{1} \cdots \Delta a_{n}, \\
\theta\left(d a_{1} \cdots d a_{n}\right)=\Delta a_{1} \cdots \Delta a_{n} .
\end{array}\right.
$$

In fact we have a bijection between such pairs $(\alpha, \Delta)$ and homorphisms: $\Omega(A) \rightarrow B$ (of grade 0 ):

$$
\Omega(A)
$$

$$
\begin{array}{lll}
\uparrow & \searrow \theta & \\
A & \stackrel{\alpha}{\rightrightarrows} & B
\end{array}
$$

This applies in particular (making $\Delta=\delta \circ \alpha$ ) to homomorphisms $\alpha: A \rightarrow B$ into $\mathbf{Z} / 2$-graded algebras $B$ possessing a graded derivation $\delta$. And the further specialization $B=\Xi,(\Xi, \delta)$ a $\mathrm{Z} / 2$-graded differential

\footnotetext{
${ }^{6}$ Operations within the free algebra are indicated by a dot.

${ }^{7}$ And also by direct proof, using the original definition (1.12) of $\Omega(A)$-this showing the identity of the original definition and the definition as a quotient.

${ }^{8} \mathrm{Of} \mathbf{Z} / 2$-graded complex algebras (in particular, of grade zero).
} 
algebra $^{9}$, then yields a functor $\Omega($ ) from the category of $\mathbf{Z} / 2$-graded algebras to that of $\mathbf{Z} / 2$-graded differential algebras:

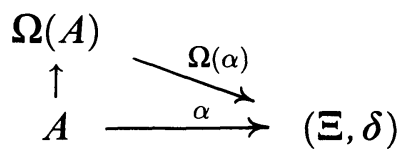

The special case where $(\boldsymbol{\Xi}, \delta)$ is bigraded, and $\alpha$ ranges in the $\mathbf{N}=0$ part of $\Xi$, then furnishes a functor from the category of $\mathbf{Z} / 2$-graded algebras to that of bigraded differential algebras.

1.2. The second kind of differentiable envelope: the differentiable algebra $\Omega A, A$ unital. Now let $A$ be a unital Z/2-graded algebra with unit 1.

Defining as follows a linear map $m: A \otimes A \rightarrow A:{ }^{10}$

$$
\begin{aligned}
& m\left(\sum_{i=1}^{k} a_{i} \otimes b_{i}\right)=\sum_{i=1}^{k}(-1)^{\partial b_{i}} a_{i} b_{i}, \\
& \quad a_{i} \in A, b_{i} \in A^{*}, i=1, \ldots, k
\end{aligned}
$$

as well as linear maps $\phi^{\perp}, \phi: A \otimes A \rightarrow A \otimes A$ :

$$
\left\{\begin{array}{l}
\phi^{\perp}(T)=m(T) \otimes \mathbf{1}, \quad T \in A \otimes A, \\
\phi=\mathrm{id}-\phi^{\perp},
\end{array}\right.
$$

we get supplementary idempotents $\phi, \phi^{\perp}$, such that

$$
\left\{\begin{array}{l}
J=\operatorname{Im} \phi=\operatorname{Ker} \phi^{\perp}=\operatorname{Ker} m, \\
K=\operatorname{Ker} \phi=\operatorname{Im} \phi^{\perp}=A \otimes \mathbf{1} .
\end{array}\right.
$$

Moreover, if one sets

$$
\left\{\begin{array}{l}
x(a \otimes b)=x a \otimes b, \\
(a \otimes b) x=a \otimes b x,
\end{array} \quad a, b, x \in A,\right.
$$

$J$ becomes a graded $A$-bimodule: therefore the direct sum

$$
\Omega A=\sum_{n \in \mathbf{N}} \Omega A^{n}, \quad\left\{\begin{array}{l}
\Omega A^{0}=A, \\
\Omega A^{n}=J \otimes_{A} J \otimes_{A} \cdots \otimes_{A} J(n \text { factors })
\end{array}\right.
$$

is a unital algebra with unit $\mathbf{1}$ (for the product $\otimes_{A}$ ), $\mathbf{N}$-graded and $\mathbf{Z} / 2$ graded for both the intrinsic grading $\partial_{0}$ (stemming from the grading of $J^{\otimes_{A} n}$ as a tensor product) and the total grading, sum of the intrinsic and the $\mathbf{N}$-grading. Further, setting

$$
d a=\mathbf{1} \otimes a-(-1)^{\partial a} a \otimes \mathbf{1}, \quad a \in A,
$$

${ }^{9}$ I.e. $\delta$ is of grade one and $\delta^{2}=0$.

${ }^{10}$ In the case of an algebra of functions $m$ is the "diagonal map" assigning to functions of two variables their values on the diagonal. 
specifies a graded derivation $d: A \rightarrow J$ :

$$
d(a b)=(d a) b \oplus(-1)^{\partial a} a d b, \quad a \in A^{*}, \quad b \in A,
$$

vanishing on the unit $\mathbf{1}$ and fulfilling

$$
a d b=a \otimes b-(-1)^{\partial b} a b \otimes \mathbf{1}=\phi(a \otimes b), \quad a, b \in A .
$$

Since $d$ uniquely extends to a differential $d$ of $\Omega A$ (i.e. a $\partial$-graded derivation of vanishing square), moreover of $\mathrm{N}$-grade $1, \Omega A$ becomes a $\mathbf{Z} / 2$-graded (in fact a bigraded) differential algebra.

As was the case above for $\Omega(A), \Omega A$ is a universal object-but now "in the unital category". $\Omega A$ could be defined as the quotient of the free algebra of the set $\{a, d a ; a \in A\}$ through the ideal generated by ${ }^{11}$

$$
\begin{cases}\lambda \cdot a+\mu \cdot b \dot{+}(\lambda a+\mu b), & \\
\lambda \cdot d a \dot{+} \mu \cdot d b \dot{-}(\lambda a+\mu b), & \left\{\begin{array}{l}
\lambda, \mu \in \mathbf{C}, \\
a \cdot b \dot{-a b},
\end{array}\right. \\
d a \cdot b \dot{+}(-1)^{\partial a} a \cdot d b \dot{-}(a b), & a \in A^{\cdot}, \quad b \in A .\end{cases}
$$

Moreover, we have universality properties analogous to those of $\Omega(A)$, (but within the unital category): to each $\mathrm{Z} / 2$-graded algebra $B$, and pair $(\alpha, \Delta)$ of a homomorphism ${ }^{8}: A \rightarrow B$ and a unital graded derivation $\alpha$-derivation $\Delta$ (i.e.

$$
\Delta \mathbf{1}=0) \text {, }
$$

there is a homomorphism $\theta: \Omega A \rightarrow B$ lifting $\alpha$, namely

$$
\left\{\begin{array}{l}
\theta\left(a_{0} d a_{1} \cdots d a_{n}\right)=\alpha\left(a_{0}\right) \Delta a_{1} \cdots \Delta a_{n}, \\
a_{0}, a_{1}, \ldots, a_{n} \in A .
\end{array}\right.
$$

If $B$ and $\alpha$ are unital, (1.28) is automatic and $\theta$ is unital. In fact, there is a bijection between such pairs $(\alpha, \Delta)$, and homomorphisms: $\Omega A \rightarrow B$ :

\section{$\Omega A$}

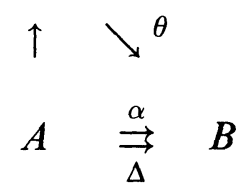

${ }^{11}$ As was the case for $\Omega(A)$, the identity of the two definitions results from the fact that both lead to the same universally property, cf. (1.31) below. Notice, on the other hand, that the second definition implies a linear bijection: $a_{0} d a_{1} \cdots d a_{n} \leftrightarrow a_{0} \otimes \hat{a}_{1} \otimes \cdots \otimes \hat{a}_{n}, \hat{a}_{i}=a_{l}(\bmod \mathbf{C} \mathbf{1})$. 
This applies (with $\Delta=\delta \circ \alpha$ ) to unital homomorphism ${ }^{8} \alpha: A \rightarrow B$, $B$ equipped with a unital graded derivation $\delta\left(\delta \mathbf{1}_{B}=0\right)$. The specialization: $B=\Xi,(\Xi, \delta)$ a unital $\mathbf{Z} / 2$ graded differential algebra (i.e. $\delta \mathbf{1}=0$ ) yields a functor from the category of unital algebras (with unital morphisms) to that of unital $\mathbf{Z} / 2$-graded differential algebras (with differential vanishing on the unit, and unital morphisms).

\section{$\Omega A$}

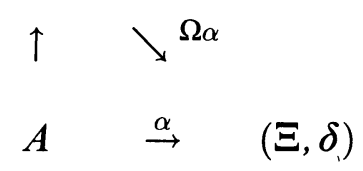

$\Omega$ induces in turn a functor from the category of unital $\mathbf{Z} / 2$-graded algebras to that of unital bigraded differential algebras, taking bigraded differential algebras $(\Xi, \delta)$ and homomorphisms $\alpha$ with range within the $n=0$ part of $\Xi$.

\section{Relationship between the two types of differential envelopes.}

2.1. The isomorphism $\tilde{\Omega}(A) \cong \Omega \tilde{A}$. Let $A$ be an arbitrary $\mathbf{Z} / 2$ graded algebra (unital or not): the differential envelope $(\tilde{\Omega}(A), d)$ of the first kind is in fact isomorphic (as a bigraded differential algebra) to the differential envelope $(\Omega \tilde{A}, d)$ of the second kind pertaining to the augmentation $\tilde{A}$ of $A^{12}$.

This fact easily follows from the above-mentioned universality properties. On the one hand (1.18) (with $\alpha=i: A \hookrightarrow \tilde{A}$, and $(\Xi, \delta)=$ $(\Omega \tilde{A}, d))$ yields a homomorphism $\Omega(i): \Omega(A) \rightarrow \Omega \tilde{A}$ with unique extension to $\tilde{\Omega}(A)$ as a unital homomorphism. On the other hand (1.31) (with $A$ replaced by $\tilde{A}, \alpha=$ id: $\tilde{A} \rightarrow \tilde{A}$ and $(\Xi, \delta)=(\tilde{\Omega}(A), d)$ ) yields a unital homomorphism $\Omega$ id $=\Omega \tilde{A} \rightarrow \tilde{\Omega}(A)$, inverse of the preceding.

As is to be expected (1.21) combined with the fact $\tilde{A}=\mathbf{C} \tilde{\mathbf{1}} \oplus A$ implies that we have:

$$
\tilde{\Omega}(A)^{1}=\tilde{A} \otimes \tilde{A} \bmod \tilde{A} \otimes \tilde{\mathbf{1}} \cong \tilde{A} \otimes A .
$$

2.2. $\Omega A$ as consisting of tensors over $A$. $A$ being now a unital $Z / 2$ graded algebra with unit 1 , we will conversely construct the differential envelope $\Omega A$ of the second type in terms of that of the first kindin fact as a subalgebra of the right ideal $A \Omega(A)=1 \Omega(A)$ of $\Omega(A)$,

\footnotetext{
${ }^{12}$ Hence we could consider the notation $\tilde{\Omega}(A)$ as superfluous, keeping the symbols $\Omega \tilde{A}$ and $\Omega(A)$ with $\Omega \tilde{A}=\mathbf{C} \tilde{\mathbf{i}}+\Omega(A)$.
} 
with the product reducing to "concatenation", and under a modified differential. We noted earlier (cf. footnote 11) the linear isomorphism

$$
\Omega A \cong \sum_{n \in \mathbf{N}}^{\oplus} A \otimes(A / \mathbf{C 1})^{\otimes n} \cong \sum_{n \in \mathbf{N}}^{\oplus} A^{\otimes n} / J_{n}
$$

with

$$
\begin{gathered}
J_{n}=A \otimes \mathbf{1} \otimes A \otimes \cdots \otimes A+A \otimes A \otimes \mathbf{1} \otimes A \otimes \cdots \otimes A \\
+\cdots+A \otimes A \otimes \cdots \otimes \mathbf{1} .
\end{gathered}
$$

This amounts in fact to quotienting $\Omega(A)$ by an ideal, the corresponding exact sequence of algebras being split, hence yielding a supplementary subalgebra of $\Omega(A)$ isomorphic to $\Omega A$.

We begin by describing these objects.

LEMMA. With a $\mathrm{Z} / 2$-graded unital, we denote by $\Delta$ the linear operator of $A \Omega(A)$ obtained by applying $d$ and projecting back in $A \Omega(A)$ :

$$
\Delta \omega=\mathbf{1} d \omega, \quad \omega \in A \Omega(A) ;
$$

and consider the direct sums

$$
K_{*}=\sum_{n=1}^{\infty}{ }^{\oplus} K_{n}
$$

where $K_{n}$ is the linear closure of elements $a_{0} d a_{1} \cdots d a_{n}$ with $a_{0}, a_{1}, \ldots$, $a_{n} \in A$ and $a_{k}=1$ for at least one $k \geq 1$; and

$$
J_{*}=\sum_{n=1}^{\infty}{ }^{\oplus} J_{n}
$$

where $J_{n} \subset A \Omega(A)^{n}$ equals

$$
J_{n}=\bigcap_{i=0}^{n-1} \operatorname{Ker} m_{i}^{n}
$$

the linear "consecutive diagonal maps" $m_{i}^{n}$ being given as follows: for $a_{0}, a_{1}, \ldots, a_{n}$

$$
\left\{\begin{array}{l}
m_{0}^{n}\left(a_{0} d a_{1} \cdots d a_{n}\right)=(-1)^{\partial a_{1}} a_{0} a_{1} d a_{2} \cdots d a_{n} \\
m_{i}^{n}\left(a_{0} d a_{1} \cdots d a_{n}\right)=(-1)^{\partial a_{t+1}} a_{0} d a_{1} \cdots d\left(a_{i} a_{i+1}\right) \cdots d a_{n}, i \geq 0 .
\end{array}\right.
$$

We then have that

(i) $K_{*}$ is the (graded) ideal of $\Omega(A)$ intersection of $A \Omega(A)$ and the ideal $\overline{K_{*}}$ of $\Omega(A)$ generated by $d \mathbf{1}$. 
(ii) $\Delta$ is a "differential modulo $K_{*}$ ", i.e. one has for $\omega, \psi \in A \Omega(A)$, $\omega$ of grade $\partial \omega$

$$
\begin{gathered}
\Delta(\omega \psi)-(\Delta \omega) \psi-(-1)^{\partial \omega} \omega \Delta \psi \in K_{*}, \\
\Delta^{2} \psi \in K_{*} .
\end{gathered}
$$

(iii) $J_{*}$ is a subalgebra of $A \Omega(A)$ on which the product reduces to "concatenation" $\times$ specified as follows ${ }^{13}$ : for $a_{0} d a_{1} \cdots d a_{n} \in J_{n}$, $b_{0} d b_{1}, \ldots, d b_{m} \in J_{m}$ :

$$
\begin{aligned}
& \left(a_{0} d a_{1} \cdots d a_{n}\right) \times\left(b_{0} d b_{1} \cdots d b_{m}\right) \\
& \quad=a_{0} d a_{1} \cdots d a_{n-1} d\left(a_{n} b_{0}\right) d b_{1} \cdots d b_{m}
\end{aligned}
$$

Note that the signs $(-1)^{\partial a_{1}}$, resp. $(-1)(-1)^{\partial a_{t+1}}$ amount to applying the linear operators $\varepsilon_{1}$, resp. $\varepsilon_{i+1}$ where $\varepsilon_{k}=\mathrm{id} \otimes \cdots \otimes \mathrm{id} \otimes \varepsilon \mathrm{id} \otimes \cdots \otimes \mathrm{id}$ with the $(k+1)$ th factor $\varepsilon$ is the grading involution of $A$ (we here use the identification (1.9)). As a consequence, leaving out these signs in the definition of $m_{0}^{n}$, resp. $m_{i}^{n}$ would not alter the definition of $J_{n}$.

Proof. (i). To prove that $K_{*}$ is an ideal it suffices to check that, given $\omega=a_{0} d a_{1} \cdots d a_{n}, a_{0}, a_{1}, \ldots, a_{n} \in A, a_{k}=1$ for $k \geq 1$, and $\psi=b_{0} d b_{1} \cdots d b_{m}, b_{0}, b_{1}, \ldots, b_{m} \in A$, one has $\psi \omega \in J_{*}$ and $\omega \psi \in J_{*}$. Now $\psi \omega \in J_{*}$ because "reordering"14 $\psi b_{0}$ does not affect the factor $d a_{k}=d 1$. To check that $\omega \psi \in J_{*}$ it obviously suffices to check that $\omega b_{0} \in J_{*}$ : this however follows from the fact that in reordering $\omega b_{0}$, the two terms not displaying a factor $d \mathbf{1}$ exactly cancel:

$$
\begin{aligned}
& \sum(-1)^{n+k-1+\sum_{i=k}^{n} \partial a_{k}} a_{0} d a_{1} \cdots d\left(a_{k-1} \mathbf{1}\right) d a_{k+1} \cdots d a_{n} d b_{0} \\
& \quad+\sum(-1)^{n+k+\sum_{i=h}^{n} \partial a_{k}} a_{0} d a_{1} \cdots d\left(a_{k-1}\right) d\left(\mathbf{1} a_{k+1}\right) d a_{n} d b_{0}=0 .
\end{aligned}
$$

We now claim that $\bar{K}_{*}$ is the linear closure of monomials $a_{0} d a_{1} \cdots d a_{n}$, $a_{0} \in \tilde{A}, a_{1}, \ldots, a_{n} \in A$ with $a_{k}=1$ for at least one $k \geq 1$. Indeed, reordering $\omega(d \mathbf{1}) \psi, \omega, \psi \in \Omega(A), \psi=x d \psi^{\prime}, x \in A$, amounts to rearranging $\omega(d \mathbf{1}) x$ whereby the factor $d \mathbf{1}$ always persists for the same reason as before. Now $K_{*} \subset A \Omega(A) \cap \bar{K}_{*}$ is obvious. And the opposite inclusion follows from the above description of $\bar{K}_{*}$ and the fact that $\omega \in A \Omega(A)$ implies $\omega=\mathbf{1} \omega$.

(ii) Check of (2.9): the l.h.s. equals

$$
\mathbf{1} d(\omega \psi)-\mathbf{1}(d \omega) \psi-(-1)^{\partial \omega} \omega \mathbf{1} d \psi=(-1)^{\partial \omega} \omega(d \mathbf{1}) \psi
$$

thus belongs to $\bar{K}_{*} \cap A \Omega(A)=K_{*}$.

${ }^{13}$ In fact the product of $\Omega(A)$ reduces to the "concatenation product" $\times$ more generally for the first factor within $J_{*}$ and the second factor any element in $A \Omega(A)$. Note, on the other hand, that $I_{1}$ and $K_{1}$ coincide via (1.9) with the respective $I$ and $K$ introduced above, cf. (1.21).

${ }^{14}$ For shortness we use the word "reordering" to mean: writing as a sum of "monomials". 
Check of (2.10): we have

$$
\Delta^{2} \psi=\mathbf{1} d(\mathbf{1} d \psi)=\mathbf{1}(d \mathbf{1}) d \psi
$$

also contained in $\bar{K}_{*} \cap A \Omega(A)=K_{*}$.

(iii) Let $\omega=a_{0} d a_{1} \cdots d a_{n}=a_{0} \otimes a_{1} \otimes \cdots \otimes a_{n} \in J_{n}$ and $\psi=$ $b_{0} d b_{1} \cdots d b_{m}=a_{0} \otimes b_{1} \otimes \cdots \otimes b_{m} \in A \Omega(A)^{m}$. Applying (1.6) and (1.5) with $x=b_{0}$ (where all but the last term vanish owing to $(2.7),(2.8)$ ) we see that the product $\omega \psi$ reduces to $(2.11)$, whose r.h.s. clearly vanishes under all maps $m_{i}^{n+m}$ for $i=1,2, \ldots, n-1$ and, if $\psi \in J_{*}$, also for $i=n, \ldots, n+m-1$.

Proposition. Let $A$ be a unital Z/2-graded complex algebra, with $\Delta, K_{*}$ and $J_{*}$ as in the last lemma. We have a split short exact sequence

$$
0 \rightarrow K_{*} \hookrightarrow A \Omega(A) \underset{\theta}{\stackrel{\pi}{\rightleftarrows}} \Omega A \rightarrow 0
$$

where ${ }^{15} \pi$ is the homomorphism of algebras, restriction to $A \Omega(A)$ of the homomorphism $\pi$ in the diagram (cf. (1.17))

$$
\Omega(A)
$$

$$
\begin{array}{lll}
\uparrow & \searrow \\
A \underset{\underline{d}}{\stackrel{\text { id }}{\rightrightarrows}} \Omega A
\end{array}
$$

i.e.

$$
\pi\left(a_{0} d a_{1} \cdots d a_{n}\right)=a_{0} \underline{d} a_{1} \cdots \underline{d} a_{n} .
$$

The lift $\theta$ of $\pi\left(\theta \circ \pi=\mathrm{id}_{\Omega A}\right)$ is the homomorphism of algebras defined by the diagram (cf. (1.30)).

\section{$\Omega A$}

$$
\begin{aligned}
& \uparrow \searrow^{\theta} \\
& A \stackrel{\text { id }}{\stackrel{D}{\rightrightarrows}} \Omega(A)
\end{aligned}
$$

i.e.

$$
\theta\left(a_{0} \underline{d} a_{1} \cdots \underline{d} a_{n}\right)=a_{0} D a_{1} \cdots D a_{n}
$$

${ }^{15}$ In the remainder of this section we will denote by $\underline{d}$ the differential of $\Omega A$ to distinguish it from the differential $d$ of $\Omega(A)$. 
where

$$
D a=\mathbf{1} d a \mathbf{1}=\mathbf{1} d a-(-1)^{\partial a} a d \mathbf{1} .
$$

One has moreover

$$
\operatorname{Im} \theta=J_{*} \quad\left(\theta\left(\Omega(A)^{n}\right)=J_{n}\right) ;
$$

consequently $\phi_{*}=\theta \circ \pi$ and $\phi_{*}^{\perp}=\mathrm{id}-\phi_{*}$ (resp. their restriction $\phi_{n}$ and $\phi_{n}^{\perp}$ to $\left.A \Omega(A)^{n}\right)$ are the projections in $A \Omega(A)\left(\right.$ resp. $\left.A \Omega(A)^{n}\right)$ corresponding to the direct sum decomposition

$$
\left\{\begin{array}{l}
A \Omega(A)=J_{*} \oplus K_{*} \\
\left(\operatorname{resp} . A \Omega(A)^{n}=J_{n} \oplus K_{n}\right) .
\end{array}\right.
$$

Finally we have the intertwining properties

$$
\begin{aligned}
& \pi \circ \Delta=\underline{d} \circ \pi, \\
& \theta \circ \underline{d}=D \circ \theta, \\
& \phi_{*} \circ \Delta=D \circ \theta .
\end{aligned}
$$

It follows in particular that if one defines

$$
D\left(a_{0} D a_{1} \cdots D a_{n}\right)=D a_{0} D a_{1} \cdots D a_{n} .
$$

$\left(J_{*}, D\right)$, with the concatenation product and the $N, \partial$ and $\partial_{0}$ gradings inherited from $A \Omega(A)$, becomes a bigraded differential algebra isomorphic to $(\Omega A, \underline{d})$.

Proof. The homomorphism $\pi$ being onto gives rise to a short exact sequence. The definition (2.17) of the homomorphism $\theta$ is justifed by the fact that $D$ is a graded derivation, vanishing on the unit, as one immediately checks:

$$
\left\{\begin{array}{l}
D(a b)=(D a) b+(-1)^{\partial a} a D b, \quad a \in A^{*}, \quad b \in A, \\
D \mathbf{1}=0 .
\end{array}\right.
$$

We note that the r.h.s. of $(2.17 \mathrm{a})$ is unchanged if one replaces throughout $D$ and $D^{\prime}$ where

$$
D^{\prime} a=d a-(-1)^{\partial a} a d \mathbf{1}=(d a) \mathbf{1} ;
$$

indeed both procedures lead to the expression

$$
a_{0}\left(d a_{1}\right) \mathbf{1}\left(d a_{2}\right) \mathbf{1} \cdots \mathbf{1}\left(d a_{n}\right) \mathbf{1},
$$

the use of $D^{\prime}$ showing that $\phi_{n}^{\perp}\left(a_{0} d a_{1} \cdots d a_{n}\right)$ is a sum of terms of the type 
(in arbitrary order, with at least one factor of the type $a_{k} d \mathbf{1}$, two such factors being never contiguous).

We now show the inclusion (hence equalities)

$$
K_{n} \subset \operatorname{Ker} \phi_{n} \subset \operatorname{Im} \phi_{n}^{\perp} \subset K_{n} .
$$

The first inclusion follows from $K_{n} \subset \operatorname{Ker} \pi$, immediate from (2.16a) since $d \mathbf{1}=0$; the second is obvious the third follows from the noted fact that $\operatorname{Im} \phi_{n}^{\perp}$ is a sum of terms of the type (2.28), where the last factor $d 1$ to the right persists whilst "reordering". Since $\phi_{n}^{2}-\phi_{n}=\phi_{n} \phi_{n}^{\perp}$, we established that $\phi_{n}$ and $\phi_{n}^{\perp}$ are idempotent.

We next show that $\operatorname{Im} \phi_{n} \subset J_{n}$ by induction. Equality holds for $n=1$. Induction step: for a monomial $\omega \in J_{n}$ and $a_{n+1} \in A$ :

$$
\omega D^{\prime} a_{n+1}=\omega \times D a_{n+1}=\omega \times\left(\mathbf{1} d a_{n}-(-1)^{\partial a_{n+1}} a_{n+1} d \mathbf{1}\right)
$$

obviously vanishes under the maps $m_{i}, i=1,2, \ldots, n$, but also under $m_{n+1}$, since

$$
m\left(x d a_{n}-(-1)^{\partial a_{n+1}} x a_{n+1} d \mathbf{1}\right)=0 .
$$

The converse inequality $\operatorname{Ker} \phi_{n}^{\perp} \supset J_{n}$ follows from the fact that for

$$
\omega=\sum_{i=1}^{k} a_{0}^{i} \cdots d a_{1}^{i} \cdots d a_{n}^{i} \in J_{n}
$$

each of the contributions to $\phi_{n}^{\perp} \omega$ corresponding to one given type of product (2.28) individually vanishes: indeed we obtain (2.28) from $a_{0} d a_{1} \cdots d a_{n}$ by replacing successively factors $d a_{k}$ of increasing $k$ by factors $a_{k} d 1$ : however, the first replacement applied to (2.20) yields zero owing to vanishing of $\omega$ under all contiguous diagonal maps.

Since (2.21) and (2.22) (hence (2.23)) immediately follow from (2.16a), (2.17a) and (2.24), we completed our proof.

To summarise we have found a variety of equivalent descriptions of the bigraded differential algebra $(\Omega A, \underline{d})$ :

- as the tensorial algebra of the $A$-bimodule $J$ under the product $\otimes_{A}$ and the differential extending (1.24);

- as linear combinations of expressions

$$
a_{0} \underline{d} a_{1} \cdots \underline{d} a_{n} \cong a_{0} \otimes \hat{a} \otimes \cdots \otimes \hat{a}_{n}, \quad a_{0}, a_{1}, a_{n} \in A,
$$

where $\hat{a}=a(\bmod \mathbf{C 1})$, and with multiplication dictated by the fact that $d$ is a differential vanishing on the unit;

- as the quotient of $A \Omega(A)$ by its ideal $K_{*}$, and $\Delta$ as a "differential modulo $K_{*}$ ", cf. (2.9), (2.10); 
- most explicitly, as the subalgebra $J_{*} \subset A \Omega(A)$, with multiplication, the "concatenation product" $\times$, and differential $D$, cf. (2.6), (2.7), (2.11), (2.18), (2.14).

3. The $Z / 2$-graded case in terms of the ungraded case. The following proposition contains two statements pertaining to the two kinds of differential envelope ${ }^{16}$.

3.1. Proposition. Let $A=A^{0} \oplus A^{1}$ be a unital $\mathbf{Z} / 2$-graded algebra (resp. a $\mathbf{Z} / 2$-graded algebra), with $\underline{A}$ the corresponding ungraded algebra ${ }^{17}$.

And consider in parallel the differential envelopes ${ }^{18}(\Omega A, d)$ (resp. $(\tilde{\Omega}(A), d))$ generated by monomials

$$
a_{0} d a_{1} \cdots d a_{n}, \quad\left\{\begin{array}{l}
a_{0} \in A(\text { resp. } \in \tilde{A}), \\
a_{1}, \ldots, a_{n} \in A,
\end{array}\right.
$$

and $(\Omega \underline{A}, d)($ resp. $(\tilde{\Omega}(\underline{A}), d))$ generated by tensors

(3.2) $a_{0} \otimes a_{1} \otimes \cdots \otimes a_{n}$,

$$
\left\{\begin{array}{l}
a_{0} \in A(\text { resp. } \in \tilde{A}), \\
a_{1}, \ldots, a_{n} \in A \bmod \mathbf{C 1}(\text { resp. } \in A),
\end{array}\right.
$$

with the product of $\Omega \underline{A}$ (resp. $\tilde{\Omega}(\underline{A})$ ) denoted by $\odot$ (hence we have, for $x \in A(\operatorname{resp} . x=\lambda \tilde{\mathbf{1}}+\underline{x} \in \tilde{A}, \lambda \in \mathbf{C}))$

$$
\left\{\begin{array}{l}
x \odot\left(a_{0} \otimes a_{1} \otimes \cdots \otimes a_{n}\right)=x a_{0} \otimes a_{1} \otimes \cdots \otimes a_{n}, \\
\left(a_{0} \otimes a_{1} \otimes \cdots \otimes a_{n}\right) \odot x \\
=\sum_{i=0}^{n}(-1)^{n+i} a_{0} \otimes \cdots \otimes a_{i} a_{i+1} \otimes \cdots \otimes a_{n} \otimes\{x(\text { resp. } \underline{x})\} \\
\quad+a_{0} \otimes a_{1} \otimes \cdots \otimes a_{n-1} \otimes a_{n} x,
\end{array}\right.
$$

where the products in the r.h.s. have to be taken $\bmod \mathbf{C 1}$ in the case of $\Omega \underline{A}$.

If we equip $\Omega \underline{A}$ (resp. $\tilde{\Omega}(\underline{A})$ ) with the new bilinear product given by

$$
S \circ T=(-1)^{m \partial_{0} S} S \odot T,
$$

where $S, T \in \Omega \underline{A}$ (resp. $\Omega(\underline{A})$ ) are respectively of intrinsic grade $\partial_{0} S$ and of $\mathbf{N}$-grade $m$, we then have that $(\Omega A, d)$ and $(\Omega \underline{A}, d)(\operatorname{resp} .(\tilde{\Omega}(A), d)$

${ }^{16}$ The second statement follows from the first via $\tilde{\Omega}(A)=\Omega \tilde{A}$.

${ }^{17}$ I.e. $\underline{A}=A$ as an algebra, with the trivial grading $\underline{A}^{0}=A, \underline{A}^{1}=\{0\}$.

${ }^{18} \Omega A$ and $\Omega \underline{A}$ (resp. $\tilde{\Omega}(A)$ and $\tilde{\Omega}(\underline{A})$ ) coincide as $\mathbf{N}_{-}, \partial_{0^{-}}$, and $\partial$-graded vector spaces. 
and $(\tilde{\Omega}(\underline{A}), d))$ are isomorphic bigraded differential algebras ${ }^{19}$, with the isomorphism given by

$$
\left\{\begin{array}{l}
i\left(a_{0} d a_{1} \cdots d a_{n}\right)=(-1)^{n \sum_{k=0}^{n} \partial a_{k}+\sum_{k \text { odd }} \partial a_{k}} a_{0} \otimes a_{1} \otimes \cdots \otimes a_{n} \\
a_{0} d a_{1} \cdots d a_{n} \in \Omega A(\text { resp. } \tilde{\Omega}(A)) \\
a_{0} \in A^{\cdot}\left(\operatorname{resp} . \tilde{A}^{\cdot}, a_{1}, \ldots, a_{n} \in A^{\cdot}\right)
\end{array}\right.
$$

REMARK. With $\Omega \in \Omega A$ (resp. $\in \Omega(A)$ ) the "monomial"

$$
\left\{\begin{array}{l}
\omega=a_{0} d a_{1} \cdots d a_{n}, \\
a_{0} \in A\left(\text { resp. } \in \tilde{A}^{*}\right), a_{1}, \ldots, a_{n} \in A^{.}
\end{array}\right.
$$

and defining

$$
\partial^{ \pm} \omega=\sum_{k_{\text {odd }}^{\text {even }}} \partial a_{k}, \quad \Delta \omega=n \partial_{0} \omega+\partial^{-} \omega,
$$

the sign factor $(-1)^{\Delta \omega}$ appearing r.h.s. of (3.5) corresponds to "extraction of the $n$ (grade one) symbols $d$ towards the left" whilst effecting the passage

$$
a_{0} d a_{1} \cdots d a_{n} \rightarrow a_{0} \otimes a_{1} \otimes \cdots \otimes a_{n}
$$

In " $Z / 2$-spirit", the map $i$ hence appears as more natural than the former identification (1.9). Note also that $\Delta \omega$ is coherently defined for monomials in $\Omega A$, since the intrinsic grade passes to the quotient $\hat{a}_{k}=a_{k} \bmod \mathbf{C 1}, a_{k} \in A$. Caution: $\Delta \omega$ is only defined for monomials, and does not make sense in general for homogeneous elements. We note that we have, for $\omega$ as in (3.6)

$$
\begin{gathered}
\Delta \omega= \begin{cases}\partial^{-} \omega & \text { for } n \text { even, } \\
\partial^{+} \omega & \text { for } n \text { odd },\end{cases} \\
\Delta(x d \omega)=\Delta \omega+(n+1) d x, \quad x \in A^{\cdot}\left(\operatorname{resp} . x \in \tilde{A}^{*}\right) \\
\left\{\begin{array}{l}
\Delta(\omega d \psi)=\Delta \omega+\Delta \psi+(m+1) \partial_{0} \omega, \\
\psi=b_{0} d b_{1} \cdots d b_{m} \in \Omega \underline{A}(\operatorname{resp} . \tilde{\Omega} \underline{A}) .
\end{array}\right.
\end{gathered}
$$

The claim in Proposition 3.1 that $(\Omega \underline{A}, d)$ (resp. $(\tilde{\Omega}(\underline{A}), d)$ ) are bigraded differential algebras results by taking $(\Omega, d)=(\Omega \underline{A}, d)$ (resp. $(\tilde{\Omega}(\underline{A}), d)$ in the following general fact.

${ }^{19} \Omega \underline{A}$ (resp. $\Omega(\underline{A})$ ) are equipped with the new product (3.4); the differential $d$ and the gradings $\partial, \partial_{0}$, remaining unchanged. Note that (3.5) defines $i$ coherently as a linear map since the sign factor r.h.s. amounts to applying the linear operators $\left\{\prod_{k=0}^{n} \varepsilon_{k}\right\}^{n} \prod_{k \text { odd }}, \varepsilon_{k}$ the operator id $\otimes \cdots \otimes$ id $\otimes \varepsilon \otimes$ id $\otimes \cdots \otimes$ id, where the $k$ th factor $\varepsilon$ is the grading involution of $A$ or $A$ $\bmod \mathbf{C 1}($ resp. of $\tilde{A}$ or $A$ ). 
LEMmA. Let $\Omega=\sum_{n \in \mathbf{N}}^{\oplus}\left(\Omega^{n}, d\right)$ be an $\mathbf{N}$-graded differential algebra, with product $\odot$, and assume the existence of an intrinsic $\mathbf{Z} / 2$-grading $\partial_{0}$ commuting with the $\mathbf{N}$-grading

$$
\left\{\begin{array}{l}
\Omega^{n}=\Omega^{n(+)}+\Omega^{n(-)} \\
\text { with }\left\{\begin{array}{l}
\Omega^{n( \pm)} \odot \Omega^{m(+)}=\Omega^{n+m( \pm)}, \\
\Omega^{n( \pm)} \odot \Omega^{m(-)}=\Omega^{n+m(\mp)},
\end{array} \quad n, m \in N,\right.
\end{array}\right.
$$

and with the differential:

$$
d \Omega^{n( \pm)} \subset \Omega^{n+1( \pm)}, \quad n \in N .
$$

Then $(\Omega, d)$ becomes a bigraded differential algebra (with total grading $\partial$ the sum of the intrinsic and the $N$-grading) under the new product . specified as follows:

$$
\left\{\begin{array}{l}
\omega \cdot \psi=(-1)^{m \partial_{0} \omega} \omega \odot \psi, \\
\omega, \psi \in \Omega, \omega \text { of intrinsic grade } \partial_{0} \omega, \psi \text { of } \mathbf{N} \text {-grade } m .
\end{array}\right.
$$

To prove this lemma, we have to check that the product - is associative and makes $d$ a $\partial$-graded derivative. Now let $\omega, \psi, \theta \in \Omega$, with $\omega, \psi$ of respective intrinsic grades $\partial_{0} \omega, \partial_{0} \psi$, and $\psi, \theta$ of respective $N$-grades $m, p$. We have on the one hand

$$
\begin{aligned}
(\omega \cdot \psi) \cdot \theta & =(-1)^{m \partial_{0} \omega}(\omega \odot \psi) \cdot \theta \\
= & (-1)^{m \partial_{0} \omega+p\left(\partial_{0} \omega+\partial_{0} \psi\right)} \omega \odot \psi \odot \theta \\
= & (-1)^{(m+p) \partial_{0} \omega} \omega \odot(\psi \cdot \theta)=\omega \cdot(\psi \cdot \theta)
\end{aligned}
$$

and on the other hand

$$
\begin{aligned}
d(\omega \cdot \psi) & =(-1)^{m \partial_{0} \omega} d(\omega \odot \psi) \\
& \left.=(-1)^{m \partial_{0} \omega}[(d \omega) \odot \psi)+(-1)^{n} \omega \odot d \psi\right] \\
& =d \omega \cdot \psi+(-1)^{n+\partial_{0} \omega} \omega \cdot d \psi .
\end{aligned}
$$

The remainder of Proposition 3.1 now follows from the fact that the map $i$ (obviously linear bijective) turns the product of $\Omega A$ (resp. $\tilde{\Omega}(A))$ into the new product of $\Omega \underline{A}(\operatorname{resp} . \tilde{\Omega}(\underline{A}))$ : we have

$$
i(\omega \psi)=i(\omega) \cdot i(\psi), \quad \omega, \psi \in \Omega A(\text { resp. } \in \tilde{\Omega}(A))
$$

which it suffices to check for $\omega$ and $\psi$ "monomials" 20 We first do

${ }^{20}$ The following argument applies to both the case of $\Omega A$ and that of $\tilde{\Omega}(A)$. In what follows $n(\omega)$ denotes the $\mathbf{N}$-grade of $\omega$. 
this for $\psi=d \psi^{\prime}, \psi^{\prime}$ a monomial: we have, with $T\left(a_{0} d a_{1} \cdots d a_{n}\right)=$ $a_{0} \otimes \cdots \otimes a_{n}$ and taking account of (3.11),

$$
\begin{aligned}
i\left(\omega d \psi^{\prime}\right) & =(-1)^{\Delta\left(\omega d \psi^{\prime}\right)} T\left(\omega d \psi^{\prime}\right) \\
& =(-1)^{\Delta\left(\omega d \psi^{\prime}\right)} T(\omega) \otimes T\left(\psi^{\prime}\right) \\
& =(-1)^{\Delta \omega+\Delta\left(d \psi^{\prime}\right)+n\left(\partial \psi^{\prime}\right) \partial_{0} \omega} T(\omega) \odot T\left(\psi^{\prime}\right) \\
& =(-1)^{n\left(d \psi^{\prime}\right) \partial_{0} \omega} i(\omega) \odot i\left(d \psi^{\prime}\right)=i(\omega) \cdot i\left(d \psi^{\prime}\right) .
\end{aligned}
$$

It remains to check (3.17) for $\psi=b d \psi^{\prime}, b \in A^{*} ; \psi^{\prime}$ a monomial, however, this boils down to the simpler case of $\psi=b$ : the knowledge of (3.17) in that case indeed allows one to infer from (3.18) and the associativity of the product - that one has

$$
\begin{aligned}
i\left(\omega b d \psi^{\prime}\right) & =i(\omega b) \cdot i\left(d \psi^{\prime}\right)=i(\omega) \cdot i(b) \cdot i\left(d \psi^{\prime}\right) \\
& =i(\omega) \cdot i\left(b d \psi^{\prime}\right) .
\end{aligned}
$$

The remaining proof of (3.17) for $\psi=b \in A^{\cdot}$ will now proceed by induction w.r.t. the $\mathbf{N}$-grade of $\omega^{21}$.

For $x, y \in A^{*}$ (resp. $x \in \tilde{A}^{\cdot}, y \in A$ ) we have

$$
\begin{aligned}
i[y(d x) b] & =i\left[y d(x b)-(-1)^{\partial x} y x d b\right] \\
& =(-1)^{\partial y} y \otimes x b-(-1)^{\partial x+\partial(y x)} y x \otimes b \\
& =(-1)^{\partial y} y \odot(\mathbf{1} \otimes x) \odot b \\
\text { (resp. } & =(-1)^{\partial y} y \odot(\tilde{\mathbf{1}} \otimes x) \odot b \\
& =i(y d x) \odot b=i(y d x) \cdot i(b)) .
\end{aligned}
$$

We now effect the induction step: assuming (2.19) to hold for $\omega$ and $\psi=b$, we check that it holds also for $\omega$ replaced by $\omega d x, x \in A^{*}$, and $\psi=b$, we have, by what precedes

$$
\begin{aligned}
i[\omega(d x) b] & =i\left[\omega d(x b)-(-1)^{\partial x} \omega x d b\right] \\
& =i(\omega) \cdot i(d(x b))-(-1)^{\partial x} i(\omega x) \cdot i(d b) \\
& =i(\omega) \cdot\left[i(d(x b))-(-1)^{\partial x} i(x d b)\right] \\
& =i(\omega) \cdot i[d(x b)]=i(\omega) \cdot i(d x) \cdot i(b) \\
& =i(\omega d x) \cdot i(b)
\end{aligned}
$$

completing our proof.

3.2. We shall now briefly show how the replacement of $(\tilde{\Omega}(A), d)$ by $(\tilde{\Omega}(\underline{A}), d)$ equipped with the product $(2.5)$ simplifies the formulae

\footnotetext{
(1.9).

${ }^{21}$ One could also perform a direct verification based on (1.5) rewritten using the identification
} 
of $\mathbf{Z}$ /2-graded cyclic cohomology 22 . We recall that the latter can be formulated using operators $\beta^{\prime}, \beta, \alpha, \gamma, \rho, \lambda, \Sigma$ on $\Omega(A)$ defined as follows [4], [5] $]^{23}$ : for $\omega \in \Omega$ of total grade $\partial \omega$, and $x \in A^{0}, a \in A$ :

$$
\begin{gathered}
\left\{\begin{array}{l}
\beta^{\prime}(\omega d x)=(-1)^{\partial \omega} \omega x, \\
\beta^{\prime}(a)=0, \quad \beta^{\prime}(d a)=a,
\end{array}\right. \\
\left\{\begin{array}{l}
\alpha(\omega d x)=(-1)^{(1+\partial x) \partial \omega} x \omega, \\
\alpha(a)=0, \quad \alpha(d a)=a, \\
\beta=\beta^{\prime}-\alpha,
\end{array}\right. \\
\left\{\begin{array}{l}
\rho(\omega d x)=(-1)^{(1+\partial x) \partial \omega}(d x) \omega, \\
\rho(a)=a, \quad \rho(d a)=a,
\end{array}\right. \\
\left\{\begin{array}{l}
\lambda(\omega d x)=(-1)^{(1+\partial x)(1+\partial \omega)} x d \omega, \\
\lambda(a)=a, \quad \lambda(d a)=0,
\end{array}\right.
\end{gathered}
$$

and, for $a_{0}=s \mathbf{1}+\underline{a}_{0} \in \tilde{A}^{\cdot}, \underline{a}_{0}, a_{1}, \ldots, a_{n} \in A^{\cdot}, s \in \mathbf{C}$,

$$
\begin{aligned}
\Sigma\left(a_{0} d a_{1} \cdots d a_{n+2}\right) & \\
= & (-1)^{\partial a_{1}} a_{0} a_{1} a_{2} d a_{3} \cdots d a_{n+2} \\
& +\sum_{i=2}^{n+1}(-1)^{\partial a_{i}}\left(a_{0} d a_{1} \cdots d a_{i-1}\right) a_{i} a_{i+1} d a_{i+2} \cdots d a_{n+2} \\
& \left.+(-1)^{n+1+\sum_{k=1}^{n+1} \partial a_{k}} s\left(a_{1} d a_{2} \cdots d a_{n+1}\right) a_{n+2}\right) .
\end{aligned}
$$

If one replaces $(\Omega(A), d)$ by $(\Omega(\underline{A}), d)$ the above operators are replaced by their images which we denote by corresponding underlined symbols:

$$
\underline{0}=i \circ 0 \circ i^{-1}
$$

and we have then, for $T \in \Omega(\underline{A})^{n}$ of intrinsic grade $\partial_{0} T$ and $x \in A$ of grade $\partial x$

$$
\begin{gathered}
\underline{\beta}^{\prime}(T \odot d x)=(-1)^{n} T \odot x, \\
\underline{\alpha}(T \odot d x)=(-1)^{n+\partial x \partial_{0} T} x \odot T, \\
\underline{\rho}(T \odot d x)=(-1)^{n+\partial x \partial_{0} T} d x \odot T, \\
\underline{\lambda}(T \odot d x)=(-1)^{n+1+\partial x \partial_{0} T} x \odot d T .
\end{gathered}
$$

${ }^{22} \mathrm{Cf}$. [1] formula $\left(\mathrm{a}^{\prime}\right),\left(\mathrm{b}^{\prime}\right)$, p. 60 , and [5] p. $47 \mathrm{a}$.

${ }^{23}$ With $\Omega(A)^{*}$ the algebraic dual of $\Omega(A)$, the cyclic cochains, resp. cyclic cocycles are the $\varphi \in \Omega(A)^{*}$ fulfilling $\varphi \circ \lambda=\varphi(\leftrightarrow \varphi \circ \gamma=\varphi \circ d=0)$, resp. $\varphi \circ \beta=\varphi \circ d=0(\leftrightarrow \varphi$ is a closed graded trace of $\Omega(A)$ ). The operator $\Sigma$ is proportional to the transpose of Conne's operator $S: S_{\varphi}=2 \pi \varphi \circ \Sigma$. 
For $a_{0}=s \mathbf{1}+\underline{a}_{0} \in \tilde{A}^{0}, \underline{a}_{0}, a_{1}, \ldots, a_{n+2} \in A^{0}, s \in \mathbf{C}$, we have then

$$
\begin{aligned}
& \underline{\beta}^{\prime}\left(a_{0} \otimes a_{1} \otimes \cdots \otimes a_{n+1}\right) \\
& \quad=\sum_{i=1}^{n}(-1)^{i} \underline{a}_{0} \otimes \cdots \otimes a_{i} a_{i+1} \otimes \cdots \otimes a_{n+1},
\end{aligned}
$$

$$
\begin{aligned}
& \underline{\alpha}\left(a_{0} \otimes a_{1} \otimes \cdots \otimes a_{n+1}\right) \\
& \quad=(-1)^{n+\partial a_{n+1}+\sum_{k=0}^{n} \partial a_{k}} a_{n+1} \otimes a_{0} \otimes \cdots \otimes a_{n},
\end{aligned}
$$

$$
\begin{aligned}
& \underline{\lambda}\left(a_{0} \otimes a_{1} \otimes \cdots \otimes a_{n+1}\right) \\
& \quad=(-1)^{n+1+\partial a_{n+1} \sum_{k=1}^{n} \partial a_{k}} a_{n+1} \otimes \underline{a}_{0} \otimes a_{1} \otimes \cdots \otimes a_{n},
\end{aligned}
$$

and

(3.37) $\underline{\Sigma}\left(a_{0} \otimes a_{1} \otimes \cdots \otimes a_{n+2}\right)$

$$
\begin{aligned}
= & \sum_{i=1}^{n+1}\left(a_{0} \otimes a_{1} \otimes \cdots \otimes a_{i-1}\right) \odot a_{i} a_{i+1} \otimes a_{i+2} \otimes \cdots \otimes a_{n+2} \\
& +(-1)^{n+1} s\left(a_{1} \otimes a_{2} \otimes \cdots \otimes a_{n+1}\right) \odot a_{n+2} .
\end{aligned}
$$

As we see the formulae (3.34) and (3.37) are free of sign factors depending upon the intrinsic grading - a notable simplification w.r.t. the formulae relative to $\beta^{\prime}$ and $\Sigma$ (cf. (1.5) and (3.28)).

Acknowledgments. The authors express hearty thanks to Professor M. Berger for the hospitality of IHES, where part of this work was completed.

\section{REFERENCES}

[1] A. Connes, Non commutative differential geometry I. The Chern Character in $K$-homology II. De Rham Cohomology and Non commutative Algebra. Pub. Math. IHES 257 (1985).

[2] M. Karoubi, Connections, courbures et classes caractéristiques en $K$-théorie algébrique, Canad. Math. Soc. Proc., Vol. 2, part 2, Part 1.19 (1982). Homologie cyclique et $K$-théorie, Astérisque no. 149, Société Mathématique de France (1987).

[3] W. Arveson, The harmonic analysis of automorphism groups, Operator algebras and applications. Proc. Symposia Pure Math. 38 part I 199 (1982).

[4] D. Kastler, Z/2-graded cohomology within the differential envelope, Contemporary Mathematics 62, 221 (1987). 
[5]

Cyclic Cohomology within the Differential Envelope; An Introduction to Alain Connes' Non Commutative Differential Geometry. Travaux en cours. Hermann, Paris (1987).

Received February 26, 1988. Research of the first author was supported in part by the U.S. Dept. of Energy under Grant no. DE-FG02-84ER40158 with Harvard University.

Centre de Physique Theorique

CNRS-LUMINY, CASE 907

F-13288, Marseille Cedex 09 (France)

Current address: R. Coquereaux

Gordon McKay Laboratory

Harvard University

Cambridge, MA 02138

USA 
